\title{
Morphologic patterns associated with BRCA1 and BRCA2 genotype in ovarian carcinoma
}

\author{
Robert A Soslow ${ }^{1}$, Guangming Han ${ }^{2}$, Kay J Park ${ }^{1}$, Karuna Garg ${ }^{1}$, Narciso Olvera ${ }^{1}$, \\ David R Spriggs ${ }^{3}$, Noah D Kauff ${ }^{3,4}$ and Douglas A Levine ${ }^{5}$ \\ ${ }^{1}$ Department of Pathology, Memorial Sloan-Kettering Cancer Center, New York, NY, USA; ${ }^{2}$ Department of \\ Pathology and Laboratory Medicine, University of Calgary, Calgary, Alberta, Canada; ${ }^{3}$ Gynecologic Medical \\ Oncology Service, Department of Medicine, Memorial Sloan-Kettering Cancer Center, New York, NY, USA; \\ ${ }^{4}$ Clinical Genetics Service, Department of Medicine, Memorial Sloan-Kettering Cancer Center, New York, \\ NY, USA and ${ }^{5}$ Gynecology Service, Department of Surgery, Memorial Sloan-Kettering Cancer Center, \\ New York, NY, USA
}

This study was undertaken with the hypothesis that certain common morphologic features of ovarian carcinomas are predictably associated with $B R C A 1$ and $B R C A 2$ deficiencies. We selected 43 high-grade serous carcinomas diagnosed at Memorial Sloan-Kettering Cancer Center that were studied as part of The Cancer Genome Atlas pilot project. In addition to 12 randomly selected nonfamilial BRCA-unassociated cases, all 31 Memorial Sloan-Kettering Cancer Center cases with BRCA1 or BRCA2 abnormality were included $(n=43)$. Slides were examined to assess tumor architecture, mitotic index, tumor-infiltrating lymphocytes (TILs), nuclear pleomorphism, necrosis, and involvement of fallopian tube epithelium. Comparing BRCA1-associated cases (BRCA1 germline mutation, $n=4, B R C A 1$ somatic mutation, $n=6, B R C A 1$ promoter methylation, $n=13$ ) with unassociated cases $(n=12)$ identified statistically significant differences in morphology. BRCA1-associated high-grade serous carcinomas had more frequent Solid, pseudoEndometrioid, and Transitional cell carcinomalike morphology (SET features) $(P=0.0045)$, higher mitotic indexes $(P=0.012)$, more TILs $(P=0.034)$, and either geographic or comedo necrosis $(P=0.034)$. BRCA2-associated cases (germline mutation, $n=4$ and somatic mutation, $n=4)$ tended to show SET features, but they were relatively deficient in TILs and necrosis. Two algorithms incorporating tumor architecture, necrosis, and either mitotic indexes or TILs separated cases that showed 2 of 3 features (BRCA1 associated) from those with 0 of 3 features (BRCA unassociated; $P=0.0016$ and $P=0.0033$ ). A test set comprising $9 B R C A 1$ germline mutants and 14 high-grade serous carcinoma controls lacking $B R C A 1$ and $B R C A 2$ germline mutation was used to validate the algorithms, with specific emphasis on the ability to detect cases with BRCA1 germline mutation. Best results were obtained with the algorithm that incorporated SET features, necrosis, and mitotic index $(P=0.0072$; sensitivity of $1.0(95 \% \mathrm{Cl}, 0.66-1.0)$; specificity of $0.57(95 \% \mathrm{Cl}, 0.29-0.82)$; positive predictive value of $0.60(95 \% \mathrm{Cl}, 0.32-0.84)$ and a negative predictive value of $1.0(95 \% \mathrm{Cl}, 0.63-1.0))$. These preliminary data indicate potential strong associations between morphology and genotype in high-grade serous carcinomas.

Modern Pathology (2012) 25, 625-636; doi:10.1038/modpathol.2011.183; published online 23 December 2011

Keywords: $B R C A$; hereditary ovarian cancer; morphology; phenotype; serous carcinoma; transitional cell carcinoma; endometrioid carcinoma; tumor infiltrating lymphocytes

High-grade serous carcinomas of the fallopian tube, ovary, and peritoneum are heterogeneous neoplasms

Correspondence: Dr RA Soslow, MD, Department of Pathology, Memorial Sloan-Kettering Cancer Center, 1275 York Avenue, MRI1027, New York, NY 10065, USA.

E-mail: gynbreast@mskcc.org

Received 1 April 2011; revised 18 October 2011; accepted 18 October 2011; published online 23 December 2011 with a variety of morphologic appearances, genotypes, chemosensitivity profiles and prognoses, despite many shared pathologic, clinical, and biologic features. Approximately $30-40 \%$ of these carcinomas exhibit BRCA1 or BRCA2 inactivation deriving from germline or somatic mutation or from methylation of the BRCA1 promoter. ${ }^{1-4}$ There is now a general consensus that most or perhaps all BRCA1-associated adnexal carcinomas are 
high-grade serous carcinomas, although the characteristics of BRCA2-mutated ovarian cancers are currently not well documented. BRCA1 inactivation may be associated with relative chemosensitivity ${ }^{3,4}$ and favorable prognosis, ${ }^{5,6}$ as compared with highgrade serous carcinomas with intact BRCA1 function, although it is not currently known whether these features are restricted to patients with the bestunderstood mechanism for $B R C A$ inactivation, $B R C A 1$ germline gene mutation. Inhibitors to polyADP ribose polymerase (PARP), which are theoretically more beneficial to patients whose tumors have deficient BRCA1 or BRCA2 function, are currently being tested in clinical trials, with positive preliminary results. $^{7-9}$ There is mounting clinical interest in predictive biomarkers to identify chemosensitive high-grade serous carcinomas that pursue a relatively favorable clinical course and might be efficiently targeted with PARP inhibitors or related agents. Morphologic criteria at the time of diagnosis would be useful in choice of treatment and patient counseling. Determining which high-grade serous carcinoma patients are more likely than others to harbor germline $B R C A 1$ or $B R C A 2$ mutations is also of great interest for selecting patients for genetic testing.

Associations between high-grade serous carcinoma morphology and prognosis and genotype have also been studied, but results are limited. Investigators have documented the relationship between the presence of tumor-infiltrating lymphocytes (TILs) and favorable prognosis, ${ }^{10-13}$ and between TILs and $B R C A$ status. ${ }^{13}$ Nearly as interesting are the classic descriptions of ovarian transitional cell carcinomas, now regarded by many to represent high-grade serous carcinoma variants, with favorable prognosis and relative chemosensitivity. ${ }^{14-16}$ We therefore questioned whether these morphologic features were associated with BRCA1 abnormality, given the associations of each with favorable prognosis and chemosensitivity. Furthermore, solid architecture, nuclear pleomorphism, geographic necrosis, high mitotic index, and TILs have been described in BRCA1-associated breast carcinomas. ${ }^{17-20}$ This study was undertaken with the hypothesis that routinely assessed morphologic features of ovarian carcinomas are reliably associated with BRCA1 and $B R C A 2$ deficiency states.

\section{Materials and methods}

\section{Training Set}

We selected 43 HGSCs diagnosed at Memorial Sloan-Kettering Cancer Center (MSKCC) that were studied as part of The Cancer Genome Atlas pilot project. ${ }^{21}$ The Cancer Genome Atlas, a multi-institutional effort sponsored by the National Cancer Institute and the National Human Genome Research Institute, catalogs genomic alterations responsible for cancer using high-throughput genome analysis methods. Techniques used include gene expression profiling, copy number assessment, SNP genotyping, genome-wide DNA methylation profiling, microRNA profiling, and whole-exome sequencing. High-grade serous carcinoma is one of the tumor types recently completed by The Cancer Genome Atlas project. ${ }^{21}$ Quality control for all The Cancer Genome Atlas specimens is accomplished through a biospecimen core resource to ensure tumor cellularity and nucleic acid integrity.

In addition to 12 randomly selected $B R C A$ unassociated cases, all MSKCC cases with BRCA abnormality were included $(n=31)$. All tumors studied were diagnosed at MSKCC as HGSC, based upon Gilks' modification to the WHO criteria for diagnosing high-grade serous carcinoma. ${ }^{22}$ Gilks' modification ${ }^{22}$ classifies high-grade ovarian carcinomas as high-grade serous carcinoma for cases showing solid, cribriform, microcystic, and transitional cell carcinoma-like architectural patterns as long as confirmatory endometrioid features such as squamous metaplasia, endometrioid adenofibroma, associated endometriosis, and synchronous endometrioid carcinoma of endometrium are absent. There were 4 cases with BRCA1 germline mutation, 13 cases with $B R C A 1$ promoter methylation, 6 cases with BRCA1 somatic mutation, 4 cases with BRCA2 germline mutation, 4 cases with BRCA2 somatic mutation, and 12 cases without these abnormalities.

Slides from the MSKCC archives were retrieved after obtaining Internal Review Board approval. The mean number of slides reviewed per case was 10 , with the minimum number of slides being 3 . Every effort was made to study tumor characteristics in the primary site (ie, fallopian tube and ovary), but omental metastases were studied if slides representing the primary site were not available. All slides were reviewed by one pathologist (RAS) without knowledge of genotype.

Slides were first examined at scanning magnification $(\times 20)$ for architectural characterization and the presence of obvious TILs, comedo-like or geographic necrosis (Figure 1), nuclear pleomorphism (Figure 2), and tubal epithelial involvement. The number of TILs per high-power field $(\times 400$; Figure 3) and the mitotic index, expressed as mitoses per 10 high-power fields, were assessed after having identified areas enriched for each characteristic at $\times 20$ magnification (see following), whenever possible.

The following architectural patterns were noted: solid (Figure 4), cribriform/pseudoendometrioid (Figure 5), transitional cell carcinoma-like (Figure 6), papillary (Figure 7), micropapillary (Figure 8), compressed micropapillary (Figure 9), infiltrative papillary (Figure 10), and infiltrative micropapillary (Figure 11). The architectural descriptions of transitional cell carcinoma- like architecture provided by Eichhorn and Young ${ }^{23}$ used to categorize these cases were undulating, diffuse, insular, trabecular, 'punched out microspaces' (the 
a

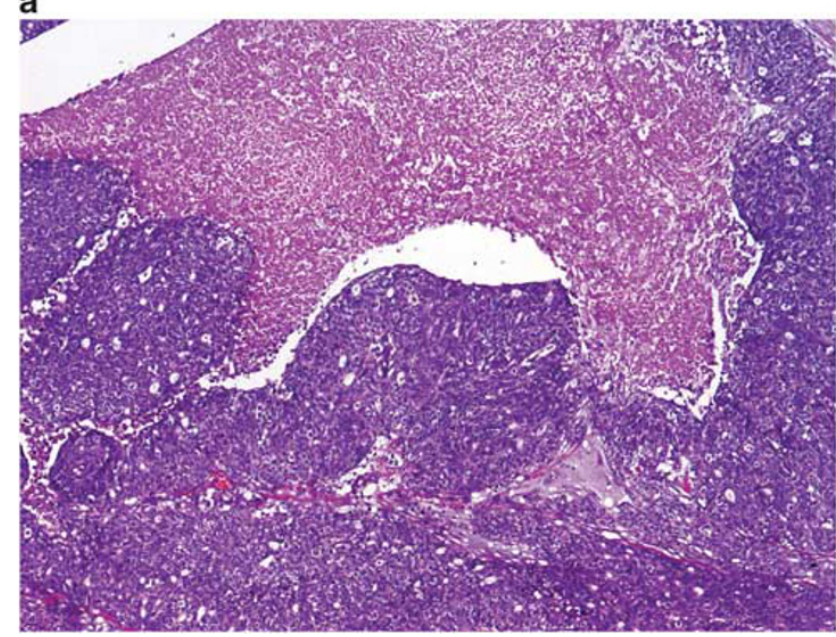

Figure 1 (a) Geographic necrosis and (b) comedo necrosis.

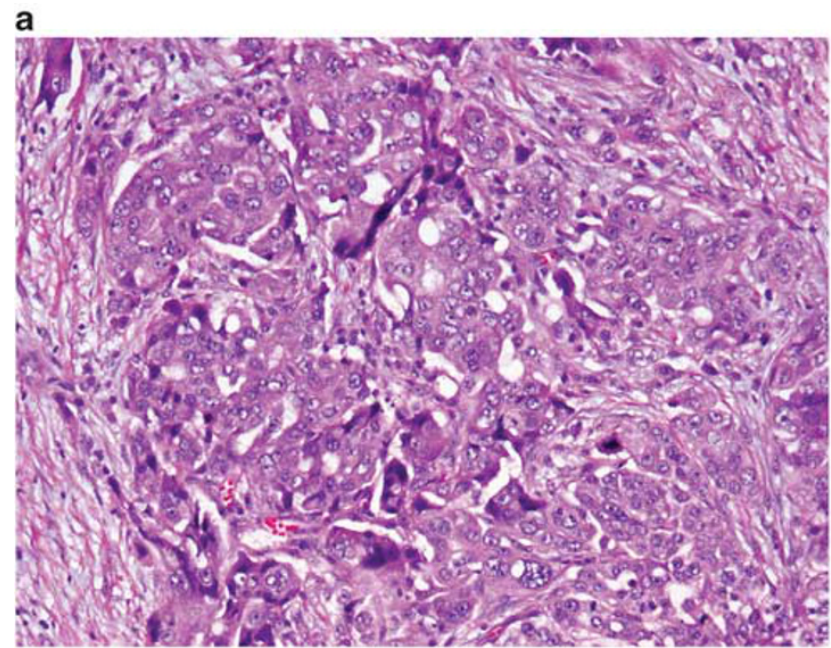

b

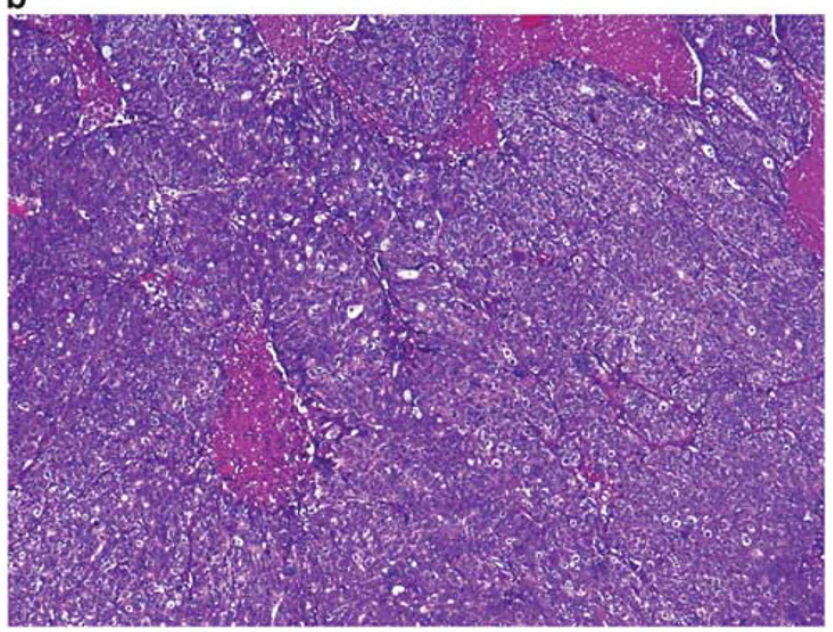

b

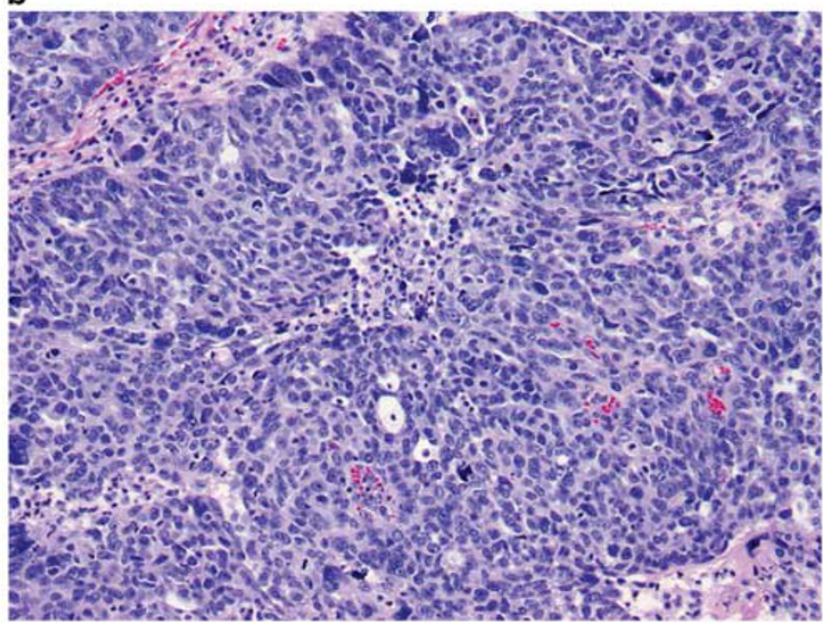

Figure 2 (a, b) Nuclear pleomorphism contrasts with areas showing small, uniform tumor cells.

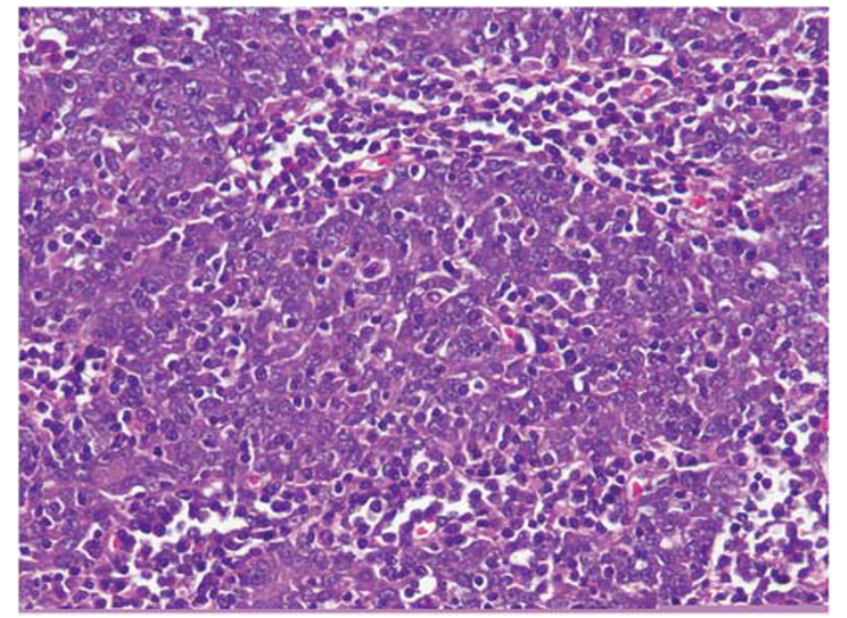

Figure 3 Tumor-infiltrating lymphocytes.

appearance of which overlaps with cribriform and pseudoendometrioid growth patterns), and large blunt papillae. The micropapillary pattern
(Figure 8) describes high-grade serous carcinoma with architectural similarity to micropapillary serous borderline tumor (also known as noninvasive low-grade micropapillary serous carcinoma). Compressed micropapillary patterns (Figure 9) were recognized when intermediate-power microscopy was required to distinguish closely spaced micropapillae, sometimes with slit-like spaces between them, from tumors with solid architecture. Tumors with destructive stromal invasion in the form of papillae with fibrovascular support were considered part of the spectrum of infiltrative papillary architecture (Figure 10) and tumors with destructive stromal invasion in the form of micropapillae lacking fibrovascular support were considered to show infiltrative micropapillae (Figure 11). The extent of each pattern was visually estimated by roughly calculating the area involved on each slide of the entire case. Necrosis and nuclear pleomorphism were scored as present when easily appreciated foci were present in the majority of slides reviewed. 


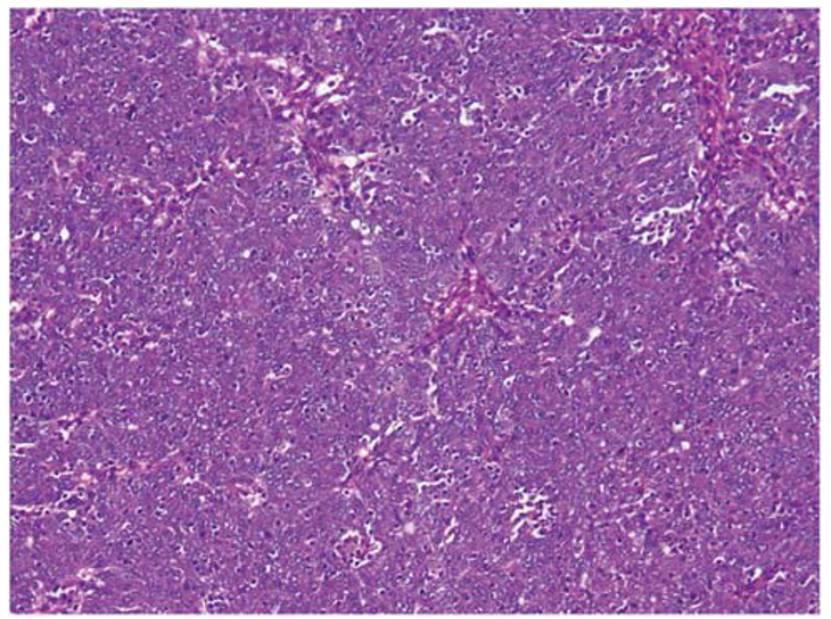

Figure 4 Solid architecture.

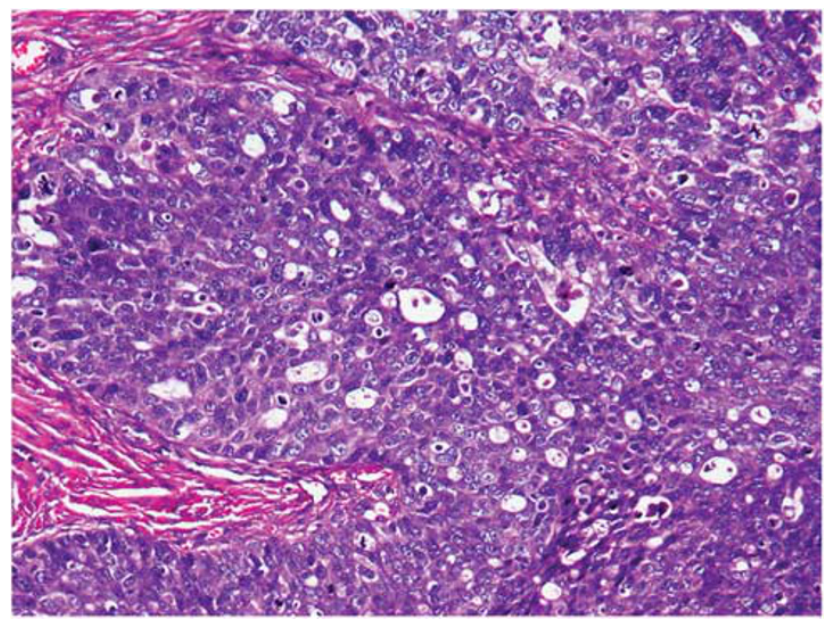

Figure 5 Cribriform/pseudoendometrioid architecture.

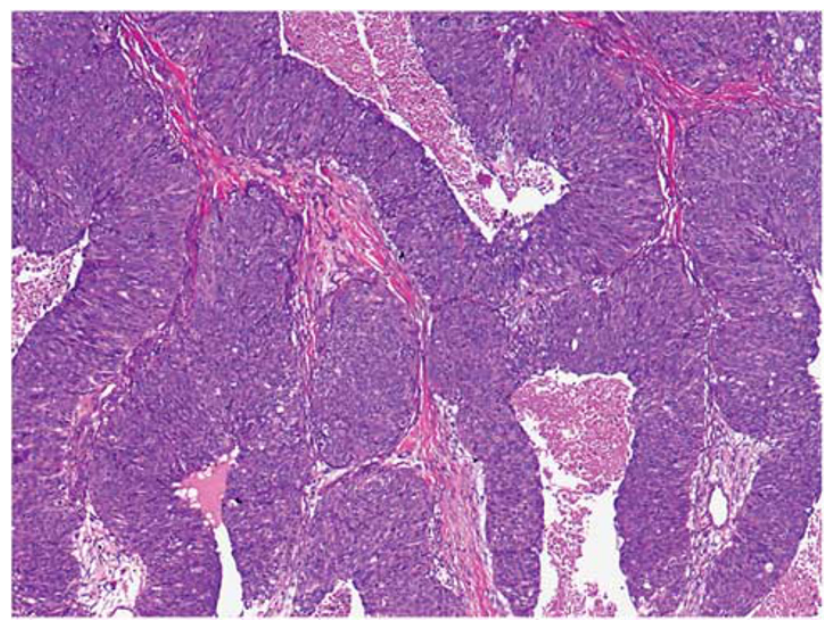

Figure 6 Transitional cell carcinoma-like (transitional cell carcinoma-like) architecture.

TIL and mitotic index counting was performed in areas enriched for each characteristic after having identified hot spots. 'Hot spots' describe areas in

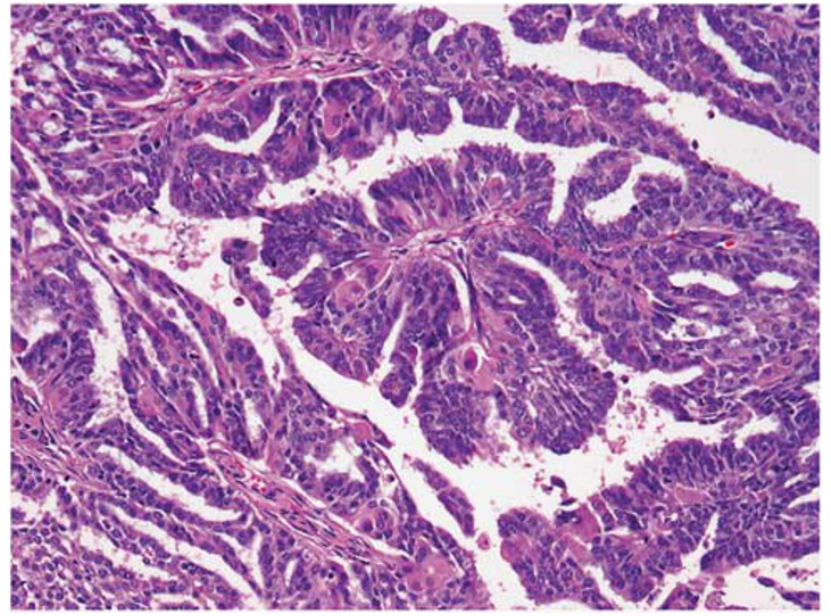

Figure 7 Papillary architecture.

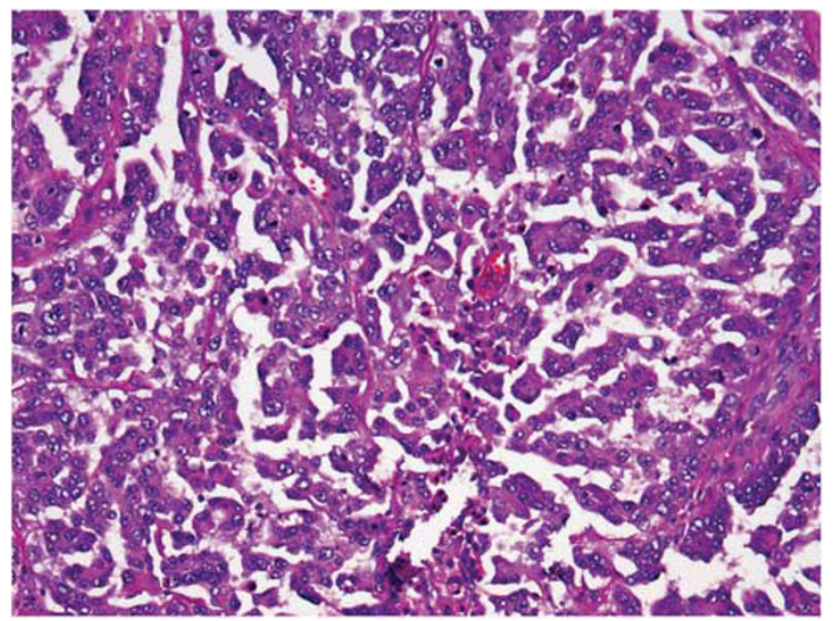

Figure 8 Micropapillary architecture.

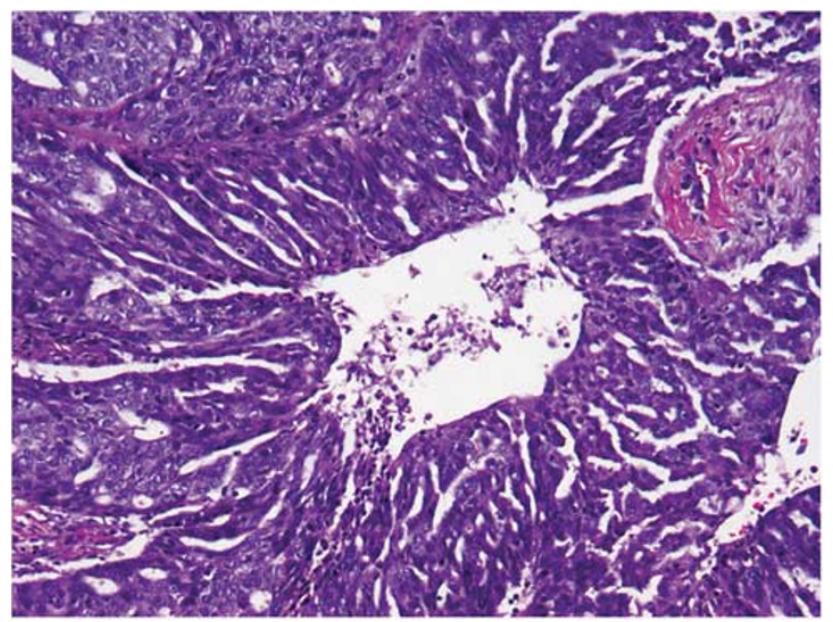

Figure 9 Compressed micropapillae.

which the highest density of TILs or mitotic figures was found at low-power magnification $(\times 20)$ after scanning all tumor slides, although it is acknowl- 


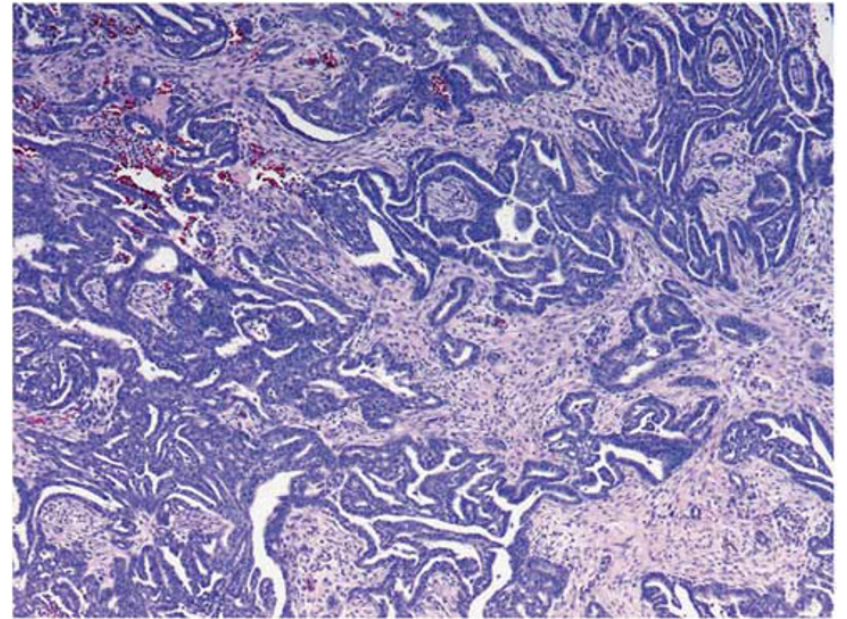

Figure 10 Infiltrative papillae.

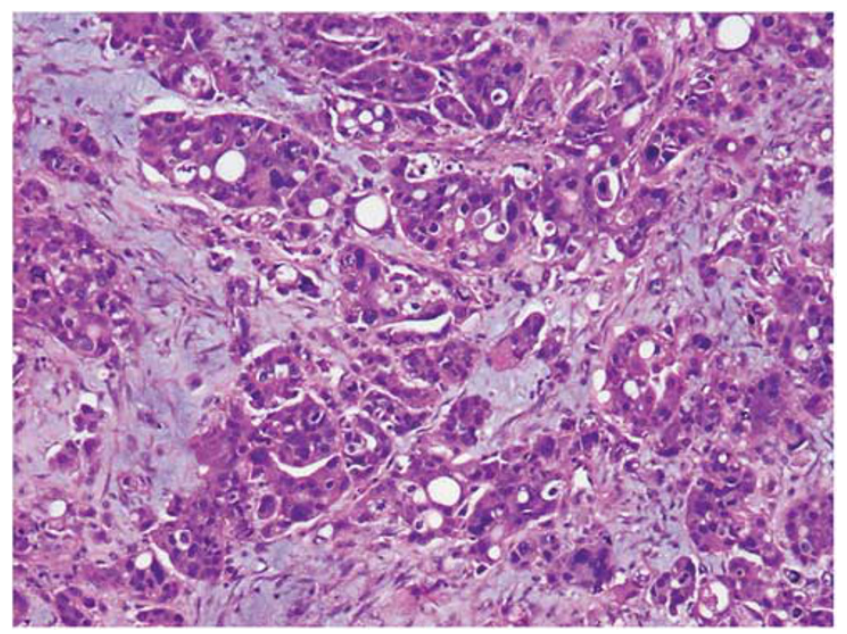

Figure 11 Infiltrative micropapillae.

edged that it was sometimes difficult to detect hot spots in cases with diffusely distributed dense TILs and high mitotic indexes or tiny hot spots in a tumor with abundant mixed acute and chronic inflammatory infiltrates that was otherwise deficient in dense TILs. Efforts at identifying hot spots revealed at least three patterns of TIL distribution: (1) diffusely dense TILs in the primary site; (2) focal TIL hot spots in a background of a TIL-poor tumor in the primary site; and (3) focal TIL hot spots most obvious in metastatic locations. For TILs, care was taken to count only intercellular lymphocytes, not lymphocytes within blood vessels or at the periphery of tumor nests. Fallopian tube epithelial involvement in the form of either serous tubal intraepithelial carcinoma or invasive high-grade serous carcinoma was scored only when sections of fimbria were available for review. Including cases without representation of fimbria might have compromised our ability to detect whether these putative precursors of high-grade serous carcinoma, usually localized to the distal one-third of the fallopian tube, including the fimbria, are differentially distributed in cases with and without mutations.

Preliminary data were then correlated with genotype. The distribution of solid, cribriform, and transitional cell carcinoma-like patterns overlapped significantly, and hence they were collapsed into one value, SET features ('Solid, pseudoEndometrioid and/or Transitional cell carcinoma-like') for subsequent calculations. Cases showing more than 25\% SET features were coded as showing SET features. When more than one SET pattern was identified, the percentage values were added together. For example, if a tumor showed $20 \%$ solid architecture and $10 \%$ pseudoendometrioid architecture, the SET value was $30 \%$ and the case was coded as SET-like. The data for BRCA1 germline and somatic mutations were combined with those of the BRCA1 promoter methylation groups for subsequent calculations involving the training set because of relative paucity of the former cases and substantial morphologic overlap between these groups. We use the term 'BRCA1-associated' to distinguish these from cases lacking BRCA1 and BRCA2 abnormalities (ie, 'BRCAunassociated' cases).

\section{Statistical Methods}

Prevalence of morphologic features was compared between the 23 cases with germline or somatic BRCA1 inactivation (including somatic mutation and promoter methylation) with the $12 B R C A$ unassociated cases. Categorical values were compared using Fisher's exact test (two tailed) and continuous variables were compared using MannWhitney test (two tailed). All statistical analyses were performed using GraphPad Prism software. Given the small number of case with BRCA2 inactivation, qualitative differences between cases with BRCA2 inactivation and both BRCA1-associated and BRCA-unassociated cases are described, but formal statistical testing was not performed.

\section{Algorithm Construction}

Morphologic features that were statistically different between the BRCA1-associated cases and the BRCAunassociated cases were used to construct two algorithms that might be useful for routine diagnostic work. Each algorithm was constructed with three variables and scored a tumor as BRCA1 related if $\geq 2$ features were present. Cases lacking any features were scored as BRCA unrelated and cases showing only one feature were considered indeterminate.

\section{Test Set}

Validation of the algorithms was then attempted using 23 additional serous ovarian cancer cases not included in The Cancer Genome Atlas that were 
Table 1 Summary of morphologic characteristics of the training set organized by genotype

\begin{tabular}{|c|c|c|c|c|c|c|}
\hline & $\begin{array}{l}\text { Tumor-infiltrating } \\
\text { lymphocytes per } \\
\text { high-power } \\
\text { field, median } \\
\text { (range) }\end{array}$ & $\begin{array}{l}\text { Mitotic } \\
\text { index, } \\
\text { median } \\
\text { (range) }\end{array}$ & $\begin{array}{c}\text { Solid, } \\
\text { pseudoendometrioid, } \\
\text { and transitional cell } \\
\text { carcinoma-like (SET) } \\
\text { features }\end{array}$ & Necrosis & $\begin{array}{c}\text { Nuclear } \\
\text { pleomorphism }\end{array}$ & $\begin{array}{c}\text { Fallopian } \\
\text { tube } \\
\text { involvement }\end{array}$ \\
\hline BRCA1 germline $(n=4)$ & $31(10-69)$ & $52(40-71)$ & $2 / 4$ & $3 / 4$ & $2 / 4$ & $1 / 1$ \\
\hline$B R C A 1$ somatic $(n=6)$ & $29(5-62)$ & $64(19-105)$ & $4 / 6$ & $3 / 6$ & $3 / 6$ & $5 / 5$ \\
\hline BRCA1 methylated $(n=13)$ & $52(13-88)$ & $60(17-102)$ & $10 / 13$ & $7 / 13$ & $7 / 13$ & $4 / 5$ \\
\hline BRCA2 germline $(n=4)$ & $19(5-43)$ & $62(8-126)$ & $4 / 4$ & $4 / 4$ & $4 / 4$ & $3 / 3$ \\
\hline BRCA2 somatic $(n=4)$ & $16(12-60)$ & $35(12-60)$ & $2 / 4$ & $0 / 4$ & $3 / 4$ & $2 / 2$ \\
\hline$B R C A$ unassociated $(n=12)$ & $29(2-140)$ & $37(15-94)$ & $2 / 12$ & $2 / 12$ & $3 / 12$ & $6 / 10$ \\
\hline$P$-value ${ }^{\mathrm{a}}$ & $0.034^{\mathrm{b}}$ & $0.012^{\mathrm{b}}$ & 0.0045 & 0.034 & 0.16 & 0.65 \\
\hline \multirow[t]{2}{*}{ Overall $(n=43)$} & 36 (mean) & 53 (mean) & $24 / 43$ & $19 / 43$ & $21 / 43$ & $21 / 26$ \\
\hline & 29 (median) & 51 (median) & & & & \\
\hline
\end{tabular}

${ }^{\mathrm{a}}$ Based on comparisons between BRCA1-associated and unassociated cases.

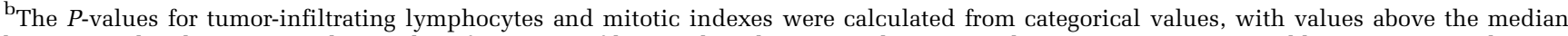
being considered 'positive.' The $P$-values for tumor-infiltrating lymphocytes and mitotic indexes as continuous variables were 0.43 and 0.030 , respectively.

'Solid, pseudoendometrioid, and transitional cell carcinoma-like' includes classically described transitional cell carcinoma-associated patterns as well as associated solid and cribriform (pseudoendometrioid) patterns (Figures 4-6). Cases showing $>25 \%$ SET were coded as showing SET features.

characterized for germline BRCA status. After excluding one case with $B R C A 2$ germline mutation, cases with and without BRCA1 germline mutations ( $n=9$ and 14, respectively) were selected from sequential patients who underwent genetic testing as part of clinical care at Memorial Sloan-Kettering Cancer Center between 2008 and 2010 (BRCA1 associated, $n=9 ; \quad B R C A$ unassociated, $n=14)$. Although all of the control case were BRCA1 wild type, it was not known whether any of these cases had BRCA1 somatic mutation, a BRCA2 somatic mutation, or methylation of the BRCA1 promoter. The mean number of slides reviewed per case in the validation set was eight, with the minimum number of slides reviewed being three. Sensitivity, specificity, positive predictive value, and negative predictive value of the algorithms were calculated using two-tailed Fisher's exact test.

\section{Results}

Table 1 displays a summary of morphologic characteristics of the training set organized by genotype.

\section{Morphologic Features of the Training Set}

Table 2 displays the distribution of architectural patterns. Of 43 cases, 8 displayed only one growth pattern (ie, a homogeneous architectural growth pattern), which was seen more commonly in BRCAassociated cases as compared with BRCA-unassociated cases. Of the 8 architecturally homogeneous cases, 7 were captured as uniformly SET in appearance (Figures 4-6). The remaining 35 cases were architecturally heterogeneous, all showing more than one growth pattern, with 8 of them showing at least 3 distinct patterns. Although patterns found in tumors from the primary site were evaluated primarily whenever possible, we noted significant morphological heterogeneity when comparing features in primary and metastatic sites. Features found more commonly in metastatic sites included infiltrative patterns with stromal desmoplasia and lymphocyterich, mixed inflammatory infiltrates at the advancing edge of infiltrative tumor nests.

The distribution of TILs (Figure 3) was also found to be relatively homogeneous in BRCA1-associated cases (pattern 1 described in Materials and Methods), whereas it tended to be heterogeneous in unassociated cases (pattern 2 described in Materials and Methods). TILs were seldom prominent at lowpower inspection of primary site tumors in BRCAunassociated cases, although hot spots could be appreciated with effort, particularly in extraovarian sites when slides representing ovary or fallopian tube contained only minimal tumor (pattern 3 described in Materials and Methods). Median TIL counts in cases with diffusely distributed TILs were 37 per high-power field.

Cases displaying obvious nuclear pleomorphism at scanning magnification contained islands of large cells, sometimes with abundant eosinophilic cytoplasm, and highly irregular nuclei, either vesicular with macronucleoli, or hyperchromatic with bizarre and convoluted nuclear forms. Psammoma bodies were less frequently seen in cases with BRCA1 abnormality (9/23 vs 10/12).

\section{BRCA1-Associated Cases}

Statistically significant associations were found between BRCA1-associated abnormality and SET 
Table 2 Architectural patterns in the training set

\begin{tabular}{|c|c|c|c|c|}
\hline $\begin{array}{l}\text { Case } \\
\text { no. }\end{array}$ & Genotype & Primary pattern & Secondary pattern & Tertiary pattern \\
\hline 1 & $B R C A 1$ germline & Solid (50\%) & Papillary $(50 \%)$ & \\
\hline $2^{\mathrm{a}}$ & BRCA1 germline & Transitional cell carcinoma (30\%) & Solid $(10 \%)$ & Infiltrating MP (10\%) \\
\hline 3 & BRCA1 germline & Papillary (100\%) & & \\
\hline 4 & $B R C A 1$ germline & Papillary $(80 \%)$ & Infiltrating MP (15\%) & Solid $(5 \%)$ \\
\hline 5 & BRCA1 methylated & Papillary $(50 \%)$ & Infiltrating papillary $(50 \%)$ & \\
\hline $6^{a}$ & BRCA1 methylated & Papillary $(50 \%)$ & Cribriform $(25 \%)$ & Solid (10\%) \\
\hline 7 & BRCA1 methylated & Cribriform $(70 \%)$ & Solid $(30 \%)$ & \\
\hline 8 & BRCA1 methylated & Infiltrating MP (50\%) & Cribriform (25\%) & Solid (25\%) \\
\hline 9 & BRCA1 methylated & Transitional cell carcinoma (100\%) & & \\
\hline 10 & BRCA1 methylated & Papillary $(60 \%)$ & MP $(35 \%$ & Solid (5\%) \\
\hline 11 & BRCA1 methylated & Transitional cell carcinoma (100\%) & & \\
\hline 12 & BRCA1 methylated & Solid (100\%) & & \\
\hline 13 & BRCA1 methylated & Solid $(50 \%)$ & Microcystic $(50 \%)$ & \\
\hline 14 & BRCA1 methylated & Solid $(50 \%)$ & Transitional cell carcinoma (50\%) & \\
\hline 15 & BRCA1 methylated & Papillary $(80 \%)$ & Solid $(20 \%)$ & \\
\hline 16 & BRCA1 methylated & Papillary $(60 \%)$ & Solid $(30 \%)$ & MP $(10 \%)$ \\
\hline 17 & BRCA1 methylated & Papillary $(80 \%)$ & MP $(15 \%)$ & Solid $(5 \%)$ \\
\hline 18 & BRCA1 somatic & Papillary $(90 \%)$ & Solid $(10 \%)$ & \\
\hline 19 & BRCA1 somatic & Papillary $(50 \%)$ & Transitional cell carcinoma (50\%) & \\
\hline 20 & BRCA1 somatic & Transitional cell carcinoma (100\%) & & \\
\hline 21 & BRCA1 somatic & Solid $(75 \%)$ & Cribriform (25\%) & \\
\hline 22 & BRCA1 somatic & Infiltrating MP (80\%) & MP $(20 \%)$ & \\
\hline 23 & BRCA1 somatic & Solid $(75 \%)$ & Cribriform (25\%) & \\
\hline 24 & BRCA2 germline & Transitional cell carcinoma (100\%) & & \\
\hline 25 & $B R C A 2$ germline & Infiltrating MP (100\%) & & \\
\hline 26 & $B R C A 2$ germline & Papillary (95\%) & Solid (5\%) & \\
\hline 27 & $B R C A 2$ germline & Transitional cell carcinoma (100\%) & & \\
\hline 28 & BRCA2 somatic & Papillary $(75 \%)$ & Infiltrating MP (25\%) & \\
\hline 29 & BRCA2 somatic & Infiltrating papillary (50\%) & Infiltrating MP $(50 \%)$ & \\
\hline 30 & BRCA2 somatic & Papillary $(50 \%)$ & Solid $(50 \%)$ & \\
\hline 31 & BRCA2 somatic & Solid $(70 \%)$ & Transitional cell carcinoma (30\%) & \\
\hline 32 & $B R C A$ unassociated & Infiltrating papillary ( $50 \%$ ) & Infiltrating MP $(50 \%)$ & \\
\hline 33 & $B R C A$ unassociated & Papillary $(60 \%)$ & MP $(30 \%)$ & Solid (10\%) \\
\hline 34 & $B R C A$ unassociated & Papillary (95\%) & Solid $(5 \%)$ & \\
\hline 35 & BRCA unassociated & Papillary $(90 \%)$ & Solid $(10 \%)$ & \\
\hline 36 & $B R C A$ unassociated & Papillary $(45 \%)$ & MP $(40 \%)$ & Solid (15\%) \\
\hline 37 & $B R C A$ unassociated & Infiltrating MP (50\%) & Papillary $(50 \%)$ & \\
\hline 38 & BRCA unassociated & Infiltrating papillary $(50 \%)$ & Infiltrating MP (50\%) & \\
\hline 39 & $B R C A$ unassociated & Infiltrating papillary $(60 \%)$ & Infiltrating MP (35\%) & Solid (5\%) \\
\hline 40 & $B R C A$ unassociated & Papillary $(65 \%)$ & Infiltrating papillary $(25 \%)$ & Solid $(10 \%)$ \\
\hline 41 & $B R C A$ unassociated & Papillary $(50 \%)$ & Cribriform (25\%) & Transitional cell carcinoma (25\%) \\
\hline 42 & $B R C A$ unassociated & Transitional cell carcinoma (100\%) & & \\
\hline 43 & $B R C A$ unassociated & Papillary $(90 \%)$ & Solid (10\%) & \\
\hline
\end{tabular}

${ }^{\mathrm{a} Q u a t e r n a r y}$ and quinary patterns were also present.

features ( $P=0.0045$; Figures 4-6). Analyzing mitotic rate as a continuous variable indicated that cases with BRCA1-associated abnormality had higher mitotic indexes than other cases $(P=0.030)$. When mitotic index was evaluated as categorical values split at the median (51), the positive association between mitotic index and BRCA1 abnormality remained significant $(P=0.012)$. Necrosis (Figure 1) was also positively associated with BRCA1 abnormality $(P=0.034)$. The TIL count was not significantly different between groups $(P=0.43)$, although it should be noted that the highest TIL counts were seen in cases with BRCA1 promoter methylation, where the median TILs count (52 per high-power field) significantly exceeded the median overall (29 per high-power field) and the median counts seen in every other group. When cases showing only focally increased TILs were excluded (patterns 2 and 3 described in Materials and
Methods), and TILs evaluated as categorical values split at the median (29 per high-power field), then the positive association between TILs and BRCA1 abnormality was significant $(P=0.034)$. Fallopian tube epithelial involvement was more frequently found in BRCA1-associated cases, but the prevalence, 9 of 10, was not significantly different from that seen in BRCA1-unassociated cases (6 of 10).

\section{BRCA2-Associated Cases}

Only eight BRCA2-associated cases were studied, four with germline mutations and four with somatic mutations. Statistical analyses were therefore not performed. However, it is notable that although these tumors as a group had a tendency to exhibit SET features (5/8 cases), none of the cases with BRCA2 somatic mutation showed geographic necrosis 


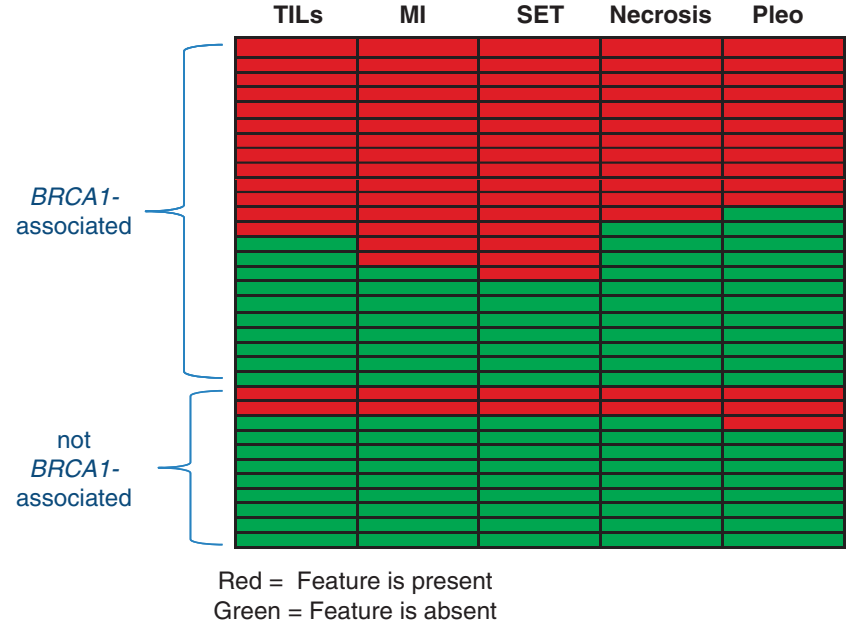

Figure 12 Heatmap showing distribution of morphologic features by genotype. Tumor-infiltrating lymphocytes, high mitotic index, solid, pseudoendometrioid, and transitional cell carcinoma-like features, pleomorphism, and necrosis are seen more frequently in BRCA1-associated cases. Green indicates feature not present, and red indicates feature present. Excluding the column indicating tubal involvement, which was not found to be discriminatory, yields a pattern with robust representation of variables (high density of red boxes; low density of green boxes) in BRCA1associated cases when compared with cases lacking BRCA-1 association (low density of red boxes; high density of green boxes). TILs: tumor-infiltrating lymphocytes >29/high-power field and diffusely distributed; MI: >51 mitotic figures per 10 high-power fields; SET: solid, pseudoendometrioid and/or transitional cell carcinoma-like architectural features in $>25 \%$ of tumors; Pleo: pleomorphism appreciable at scanning magnification in $>25 \%$ of tumors; Tubal: high-grade serous carcinoma involves tubal epithelium, including serous tubal intraepithelial carcinoma; Necrosis: necrosis appreciable at scanning magnification in $>25 \%$ of tumors; $B R C A 1$-associated includes cases with germline or somatic mutation or BRCA1 promoter methylation.

or TIL counts that exceeded the median. All evaluable cases showed involvement of fallopian tube epithelium.

\section{Algorithm}

The distribution of features seen in each case is displayed in a heatmap (Figure 12). The four variables showing statistically significant differences between BRCA1-associated and BRCA-unassociated tumors were used to devise two algorithms, each with three variables. Features evaluated in the first algorithm included SET appearance, necrosis, and diffusely distributed TILs that exceeded the median ( $>37$ TILs in one high-power field). In the second algorithm, TILs were replaced with mitotic index (mitotic index that exceeded the median ( $>51$ mitotic figures per 10 high-power field)). The algorithms scored a tumor as BRCA related if $\geq 2$ features were present. Cases lacking any features were scored as BRCA unrelated and cases showing only one feature were considered indeterminate. Tables 3 and 4 display the distribution of cases from The Cancer Genome Atlas set with and without
Table 3 Distribution of solid, pseudoendometrioid, and transitional cell carcinoma-like (SET) features, necrosis, and tumorinfiltrating lymphocytes (algorithm 1)

\begin{tabular}{lcccc}
\hline & $\begin{array}{c}\text { No } \\
\text { features }\end{array}$ & $\begin{array}{c}\text { One } \\
\text { feature }\end{array}$ & $\begin{array}{c}\text { Two } \\
\text { features }\end{array}$ & $\begin{array}{c}\text { Three } \\
\text { features }\end{array}$ \\
\hline $\begin{array}{l}\text { BRCA1 germline } \\
(n=4)\end{array}$ & 0 & 2 & $1^{\mathrm{a}}$ & $1^{\mathrm{a}}$ \\
$\begin{array}{l}\text { BRCA1 somatic }(n=6) \\
\begin{array}{l}\text { BRCA1 methylated } \\
(n=13)\end{array}\end{array}$ & 2 & 0 & $2^{\mathrm{b}}$ & $2^{\mathrm{b}}$ \\
$\begin{array}{l}\text { BRCA unassociated } \\
(n=12)\end{array}$ & 5 & $3^{\mathrm{b}}$ & $6^{\mathrm{c}}$ & $3^{\mathrm{d}}$ \\
\hline
\end{tabular}

${ }^{\mathrm{a}}$ One case with SET features.

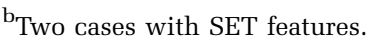

${ }^{\mathrm{c}}$ Five cases with SET features.

$\mathrm{d}_{\text {Three cases with SET features. }}$

This algorithm was tested to determine whether BRCA1 association could be predicted by the presence of two or three variables: SET features, tumor-infiltrating lymphocytes, and necrosis. The comparison group comprises cases without solid, pseudoendometrioid, and transitional cell carcinoma-like features, tumor-infiltrating lymphocytes, or necrosis. The 'one feature' column indicated cases indeterminate for BRCA1 association based on the presence of only one variable.

Table 4 Distribution of solid, pseudoendometrioid, and transitional cell carcinoma-like (SET) features, necrosis, and mitotic index (algorithm 2)

\begin{tabular}{lcccc}
\hline & $\begin{array}{c}\text { No } \\
\text { features }\end{array}$ & $\begin{array}{c}\text { One } \\
\text { feature }\end{array}$ & $\begin{array}{c}\text { Two } \\
\text { features }\end{array}$ & $\begin{array}{c}\text { Three } \\
\text { features }\end{array}$ \\
\hline $\begin{array}{l}\text { BRCA1 germline } \\
(n=4)\end{array}$ & 0 & 1 & $2^{\mathrm{a}}$ & $1^{\mathrm{a}}$ \\
$\begin{array}{l}\text { BRCA1 somatic }(n=6) \\
\text { BRCA1 methylated } \\
(n=13)\end{array}$ & 1 & 1 & $1^{\mathrm{a}}$ & $3^{\mathrm{b}}$ \\
$\begin{array}{l}\text { BRCA unassociated } \\
(n=12)\end{array}$ & 5 & 5 & $2^{\mathrm{a}}$ & 0 \\
\hline
\end{tabular}

${ }^{\mathrm{a}}$ One case with SET features.

${ }^{\mathrm{b}}$ Three cases with SET features.

${ }^{\mathrm{c}}$ Seven cases with SET features.

$\mathrm{d}_{\text {Two cases with SET features. }}$

This algorithm was tested to determine whether BRCA1 association could be predicted by the presence of two or three variables: solid, pseudoendometrioid, and transitional cell carcinoma-like features, mitotic index, and necrosis. The comparison group comprises cases without solid, pseudoendometrioid, and transitional cell carcinomalike features, mitotic index, or necrosis. The 'one feature' column indicates cases indeterminate for BRCA1 association based on the presence of only one variable.

BRCA1 abnormality when characterized according to each of these two algorithms.

Of 23 informative cases, 15 met BRCA1-associated criteria in algorithm 1 , as compared with 2 of 12 informative cases without BRCA1 abnormality $(P=0.012)$. The sensitivity of the algorithm was $88 \%(95 \%$ CI, 0.64-0.99) and the specificity was $56 \%$ (95\% CI, 0.31-0.78). Of 23 cases, 17 met $B R C A 1$-associated criteria in algorithm 2, as com- 


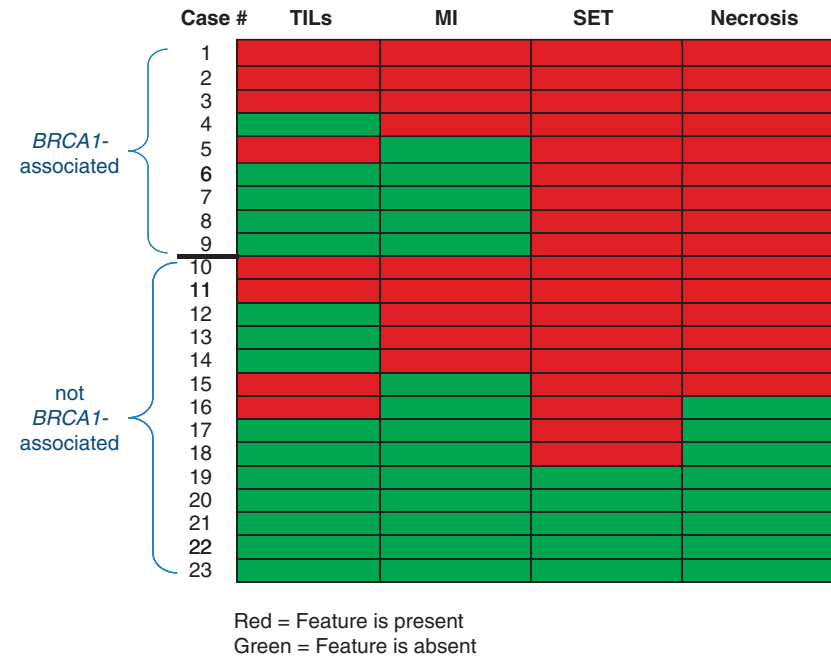

Figure 13 Heatmap of test set showing distribution of morphologic features by genotype.

pared with 2 of 12 informative cases without BRCA1 abnormality $(P=0.0033)$. The sensitivity of this algorithm was $89 \%$ (95\% CI, 0.67-0.99) and the specificity was $63 \%$ (95\% CI, 0.35-0.85).

\section{Test Set}

We were able to assemble a modest number of cases to attempt validation of these algorithms, with focus on BRCA1 germline mutation. The test set included cases with and without known BRCA1 germline mutation ( $n=9$ and 14, respectively) (Figure 13). Although none of the cases in the test set had a germline BRCA2 mutation, unlike the learning set, it was not known whether these cases had BRCA1 somatic mutation, a BRCA2 somatic mutation, or methylation of the BRCA1 promoter. As previously, cases with 2 or 3 features present were scored as 'predictive of BRCA1.' In one calculation, cases with 0 or 1 criterion were scored as 'not predictive of $B R C A 1$ ' and in a subsequent calculation, only cases with 0 features were scored as 'not predictive of BRCA1.' Cases with only one criterion were not included in the second calculation because they were deemed 'indeterminate' in the learning set.

In the first algorithm, including SET features, necrosis, and TILs, all 9 BRCA1 germline mutants showed features predictive of BRCA1; 4 cases showed all 3 features and 5 showed 2 features. This contrasted with the BRCA controls, of which 7 of 14 showed features predictive of BRCA1 (3 cases with 3 features and 4 cases with 2 features) and 7 cases showed only 1 feature, such as SET $(n=2)$, or none $(n=5)$. In the second algorithm, including SET features, necrosis, and mitotic index, all 9 BRCA1 germline mutants showed features predictive of BRCA1; 4 cases showed all 3 features and 5 showed 2 features. This contrasted with the BRCA controls, of which 6 of 14 showed features predictive of
BRCA1 (5 cases with 3 features and 1 case with 2 features) and 8 cases showed only 1 feature, such as SET $(n=3)$, or none $(n=5)$.

The first algorithm predicted BRCA1 association markedly better than chance; $P=0.019$ scoring cases with 0 or 1 feature as 'not predictive of BRCA1' and $P=0.045$ using the second calculation that omitted cases with only 1 feature. Calculations that considered cases with 0 or 1 feature as 'not predictive of BRCA1' yielded a sensitivity of 1.0 (95\% CI, 0.66-1.0); specificity of 0.50 (95\% CI, $0.23-0.77$ ); positive predictive value of 0.56 (95\% CI, $0.30-$ 0.80 ); and a negative predictive value of 1.0 (95\% CI, 0.59-1.0). Calculations that omitted cases with only one feature yielded a sensitivity of 1.0 (95\% CI, 0.66-1.0); specificity of 0.42 (95\% CI, $0.15-0.72$ ); positive predictive value of 0.56 (95\% CI, $0.30-$ $0.80)$; and a negative predictive value of $1.0(95 \%$ CI, 0.48-1.0).

The second algorithm yielded statistically significant results; $P=0.0072$ scoring cases with 0 or 1 feature as 'not predictive of BRCA1' and $P=0.038$ using the second calculation that omitted cases with only 1 feature. Calculations that considered cases with 0 or 1 feature as 'not predictive of BRCA1' yielded a sensitivity of 1.0 (95\% CI, 0.66-1.0); specificity of 0.57 (95\% CI, 0.29-0.82); positive predictive value of 0.60 (95\% CI, 0.32-0.84); and a negative predictive value of 1.0 (95\% CI, 0.63-1.0). Calculations that omitted cases with only one feature yielded a sensitivity of 1.0 (95\% CI, 0.661.0); specificity of 0.45 (95\% CI, 0.17-0.77); positive predictive value of 0.60 (95\% CI, 0.32-0.84); and a negative predictive value of 1.0 (95\% CI, 0.48-1.0).

\section{Discussion}

Comparatively little is known about relationships between high-grade serous carcinoma morphology and genotype. Although most BRCA1 germline mutation-associated ovarian carcinomas have been reported to be high-grade serous carcinomas, a relatively small number has been diagnosed as undifferentiated, high-grade endometrioid, mixed epithelial, or mucinous. ${ }^{24-26}$ Among the cases categorized as high-grade serous carcinomas, TILs (particularly CD8-positive T cells) have been recognized as characteristic only recently. ${ }^{13}$ Little or nothing is currently known about the morphology of high-grade serous carcinomas with BRCA1 somatic mutations, BRCA1 promoter methylation, BRCA2 germline mutations, ${ }^{27}$ or BRCA2 somatic mutations.

Our findings indicate a characteristic morphologic appearance of HGSCs with BRCA1 abnormality. As compared with cases lacking BRCA abnormalities, the former cases are significantly more frequently SET in appearance, contain necrosis and more TILs, and have a higher mitotic index. Obvious pleomorphism was a characteristic feature of 
BRCA abnormality, although it did not reach statistical significance. Similar morphological patterns have been described as well in BRCA1-associated triple-negative breast carcinomas, including those with a medullary or atypical medullary-like appearance. ${ }^{17-20}$ HGSCs with BRCA abnormality are also more frequently morphologically homogeneous (ie, show SET features uniformly throughout the tumor and uniformly distributed TILs) as compared with other cases, in which combinations of architectural patterns were more common.

Substantial intratumoral heterogeneity complicated derivation of criteria that might be useful in clinical practice. The extent of transitional cell carcinoma-like features, pleomorphism, and necrosis was empirically derived for segregation into 'positive' and 'negative' cases based on visual estimation, whereas 'positive' TILs and mitotic index were initially scored based on a value that exceeded the median. Because of the observation that TIL distribution tended to be homogeneous in BRCAassociated cases and heterogeneous in unassociated cases, only cases with diffusely distributed TILs with more than the median TILs per high-power field were considered TIL positive in construction of the first algorithm, as discussed subsequently. Although additional studies will be necessary to validate these specific numbers, we are confident that tumors with $B R C A$-associated abnormalities do show significantly increased TILs and mitotic indexes relative to other high-grade serous carcinomas. Another complicating factor was the frequent presence of morphological patterns and TILs in peritoneal metastases that were not present in the primary site. Analyses were restricted to primary site tumors and our statistically significant findings support that practice. It is possible that increased TILs in BRCA1unassociated cases and in metastatic sites are B cells or non-CD8-positive T cells.

We explored the application of two algorithms that took into account multiple morphological features because evaluation of only one feature for categorization into BRCA1-associated or -unassociated groups suggested this approach would be insufficient for diagnostic testing. The algorithms, devised from the learning set, separated cases showing two or three characteristic features from those with only one feature or none. Despite the apparent strengths of the algorithms, we found 2 of 12 BRCA1-unassociated cases from the training set that met criteria in both algorithms tested. It is possible that such cases represent examples of homologous recombination deficiency unrelated to specific alterations of BRCA1 or BRCA2. Homologous recombination refers to one of several mechanisms for the repair of deleterious double-strand DNA breaks. Both BRCA1 and BRCA2 gene products can accomplish this type of repair; however, there are a number of other pathways that can theoretically lead to homologous recombination. The two cases with morphologic appearances consistent with
$B R C A$ dysfunction without known BRCA defects may represent tumors containing a separate mechanism leading to homologous recombination deficiency.

We attempted to validate these algorithms in a relatively small test set with particular focus on the ability to detect cases with BRCA1 germline mutation. The strongest results derived from use of the second algorithm, which accounted for SET features, necrosis, and mitotic index. These results should be interpreted in the context of current clinical practice and knowledge regarding the prevalence of $B R C A$ abnormalities in unselected cohorts of high-grade serous carcinoma patients. Results from The Cancer Genome Atlas indicate that as many as $50 \%$ of highgrade serous carcinomas may have abnormalities in the homologous recombination pathway, with $\sim 20 \%$ having germline or somatic mutations in BRCA1 or BRCA2 somatic mutations; $11 \%$ having $B R C A 1$ promotor methylation; and an additional $20 \%$ having other abnormalities such as EMSY amplification or mutation, PTEN deletion, or mutations in one of the Fanconi Anemia genes. ${ }^{21}$ Detecting which patients have an inherited predisposition to breast and ovarian cancer typically centers on diagnosis of high-grade serous carcinoma, family history, and referral for genetic testing. Excluding SET pattern tumors from the family of high-grade serous carcinoma might prevent affected patients from eligibility for screening programs. In most centers, selection for genetic testing is individualized, although genetic testing is increasingly being offered to all high-grade serous carcinoma patients in some centers, including Memorial Sloan-Kettering Cancer Center. This approach assumes a pretest probability of BRCA1 mutation of $\sim 16-17 \%$, resulting in testing 6 patients to detect 1 with a germline BRCA1 mutation. The theoretical practical benefit of our algorithms is that because of very high negative predictive values, they exclude up to one-third of patients from the need for genetic testing, although careful evaluation of the family history remains imperative to minimize false negatives.

The data suggest that the algorithms significantly enrich the pool of patients to be tested by increasing the positive predictive value from that expected by chance (16-17\%) to as high as $60 \%$, although it is acknowledged that the test set overrepresents the prevalence of BRCA1 germline mutations. We know from the training set that SET features, necrosis, high mitotic index, and dense TILs are not restricted to tumors with BRCA1 germline mutation, such that some of the $50 \%$ of tumors meeting criteria but lacking $B R C A 1$ germline mutation will show instead BRCA1 somatic mutation, BRCA1 promoter methylation, BRCA2 somatic mutation, or, possibly, other homologous recombination abnormalities. It is unknown if the clinical features of high-grade serous carcinomas associated with BRCA1 germline mutation can be extrapolated to these different genomic settings. 
The presence of SET patterns theoretically accounts for previous reports of high-grade endometrioid, transitional cell carcinoma, and undifferentiated carcinomas in BRCA1 germline cases. ${ }^{28-30}$ Whether tumors exhibiting these architectural patterns should be classified as high-grade serous carcinoma is controversial. Immunohistochemical data demonstrate that tumors reported as ovarian transitional cell carcinomas are not only immunophenotypically dissimilar from urothelial carcinomas of the urinary tract, but also express WT1 at rates that are nearly identical to typical high-grade serous carcinoma ${ }^{31}$ Furthermore, the classical description of ovarian transitional cell carcinomas, provided by Eichhorn and Young, ${ }^{23}$ includes tumors with foci that are indistinguishable from high-grade serous carcinomas. Immunohistochemical data linking pseudoendometrioid tumors to high-grade serous carcinomas are currently lacking, but gene expression data indicate that most so-called 'highgrade ovarian endometrioid adenocarcinomas' have profiles that both overlap with high-grade serous carcinomas and vary significantly from low-grade ovarian endometrioid adenocarcinomas. ${ }^{32}$ The groups from Memorial Sloan-Kettering Cancer Center $^{33}$ and Vancouver General Hospital ${ }^{22}$ have argued in favor of subsuming SET tumors into the highgrade serous carcinoma family, and investigators have demonstrated both improved diagnostic reproducibility and the clinical relevance of this practice. $^{22,34}$ Revised Gynecologic Oncology Group criteria now regard SET tumors as high-grade serous carcinoma. Considering SET tumors as high-grade serous carcinoma variants also strongly anchors these tumors into the family of high-grade serous carcinomas, which may trigger genetic testing for BRCA abnormality. Nonetheless, we do believe that SET tumors are biologically and clinically distinctive, such that it is reasonable to call attention to their existence in diagnostic reporting (ie, highgrade serous carcinoma, transitional cell carcinomalike variant).

The number of BRCA1 mutation cases in the training set was too small to detect differences between them and cases with BRCA1 promoter methylation. Similarly, the number of cases with BRCA2 abnormality was limited in this series, but we can make some qualitative observations. Cases with BRCA1 promotor methylation had among the highest TIL counts and frequently showed SET features and high mitotic indexes. BRCA2 germline mutated cases had morphological features that overlapped with the BRCA1 group, including SET features, pleomorphism, and necrosis, although they less frequently showed high numbers of TILs. BRCA2 somatically mutated cases tended to lack TILs and necrosis, although they also retained the characteristic architecture and pleomorphism seen in BRCA1 cases.

In summary, we have demonstrated very strong correlations between genotype and high-grade serous carcinoma phenotype. Tumors lacking the characteristic histological features described herein are quite unlikely to be associated with BRCA1 abnormality. Once validated, these data may potentially be exploited to narrow the pool of high-grade serous carcinoma patients tested for germline mutations. The data also allow for recognition of tumors with potentially favorable clinical profiles that might be targeted with specific therapeutic agents. Last, our data firmly establish relationships between high-grade serous carcinomas with solid, pseudoendometrioid, and transitional cell carcinoma-like morphological features (SET features). Excluding SET pattern tumors from the family of high-grade serous carcinoma might prevent affected patients or their families from being eligible for targeted treatment or screening programs.

\section{Acknowledgement}

We thank Drs Natasha Rekhtman, Venkatraman Seshan, and Boris Bastian for their valuable guidance in preparation of this manuscript, and the Project Hope for Ovarian Cancer Research and Education for funding of this research.

\section{Disclosure/conflict of interest}

The authors declare no conflict of interest.

\section{References}

1 Risch HA, McLaughlin JR, Cole DEC, et al. Prevalence and penetrance of germline BRCA1 and BRCA2 mutations in a population series of 649 women with ovarian cancer. Am J Hum Genet 2001;68:700-710.

2 Press JZ, De Luca A, Boyd N, et al. Ovarian carcinomas with genetic and epigenetic BRCA1 loss have distinct molecular abnormalities. BMC Cancer 2008;8:17.

3 Husain A, He G, Venkatraman ES, et al. BRCA1 upregulation is associated with repair-mediated resistance to cis-diamminedichloroplatinum(II). Cancer Res 1998;58:1120-1123.

4 Yuan SS, Lee SY, Chen G, et al. BRCA2 is required for ionizing radiation-induced assembly of Rad51 complex in vivo. Cancer Res 1999;59:3547-3551.

5 Ben David Y, Chetrit A, Hirsh-Yechezkel G, et al. Effect of BRCA mutations on the length of survival in epithelial ovarian tumors. J Clin Oncol 2002;20: 463-466.

6 Boyd J, Sonoda Y, Federici MG, et al. Clinicopathologic features of BRCA-linked and sporadic ovarian cancer. JAMA 2000;283:2260-2265.

7 Audeh MW, Carmichael J, Penson RT, et al. Oral poly(ADP-ribose) polymerase inhibitor olaparib in patients with BRCA1 or BRCA2 mutations and recurrent ovarian cancer: a proof-of-concept trial. Lancet 2010;376:245-251.

8 Tutt A, Robson M, Garber JE, et al. Oral poly(ADP-ribose) polymerase inhibitor olaparib in patients with BRCA1 or BRCA2 mutations and advanced breast cancer: a proofof-concept trial. Lancet 2010;376:235-244. 
9 Fong PC, Yap TA, Boss DS, et al. Poly(ADP)-ribose polymerase inhibition: frequent durable responses in BRCA carrier ovarian cancer correlating with platinum-free interval. J Clin Oncol 2010;28:2512-2519.

10 Sato E, Olson SH, Ahn J, et al. Intraepithelial CD8+ tumor-infiltrating lymphocytes and a high CD8+/ regulatory $\mathrm{T}$ cell ratio are associated with favorable prognosis in ovarian cancer. Proc Natl Acad Sci USA 2005;102:18538-18543.

11 Zhang L, Conejo-Garcia JR, Katsaros D, et al. Intratumoral T cells, recurrence, and survival in epithelial ovarian cancer. N Eng J Med 2003;348:203-213.

12 Hamanishi J, Mandai M, Iwasaki M, et al. Programmed cell death 1 ligand 1 and tumor-infiltrating CD8+ T lymphocytes are prognostic factors of human ovarian cancer. Proc Natl Acad Sci USA 2007;104:3360-3365.

13 Clarke B, Tinker AV, Lee CH, et al. Intraepithelial T cells and prognosis in ovarian carcinoma: novel associations with stage, tumor type, and BRCA1 loss. Mod Pathol 2009;22:393-402.

14 Robey SS, Silva EG, Gershenson DM, et al. Transitional cell carcinoma in high-grade high-stage ovarian carcinoma. An indicator of favorable response to chemotherapy. Cancer 1989;63:839-847.

15 Gershenson DM, Silva EG, Mitchell MF, et al. Transitional cell carcinoma of the ovary: a matched control study of advanced-stage patients treated with cisplatin-based chemotherapy. Am J Obstet Gynecol 1993;168:1178-1187.

16 Kommoss F, Kommoss S, Schmidt D, et al. Survival benefit for patients with advanced-stage transitional cell carcinomas vs. other subtypes of ovarian carcinoma after chemotherapy with platinum and paclitaxel. Gynecol Oncol 2005;97:195-199.

17 Tisserand P, Fouquet C, Barrois M, et al. Lack of HIN-1 methylation defines specific breast tumor subtypes including medullary carcinoma of the breast and BRCA1-linked tumors. Cancer Biol Ther 2003;2: 559-563.

18 Armes JE, Egan AJM, Southey MC, et al. The histologic phenotypes of breast carcinoma occurring before age 40 years in women with and without BRCA1 or BRCA2 germline mutations: a population-based study. Cancer 1998;83:2335-2345.

19 Lakhani SR, Gusterson BA, Jacquemier J, et al. The pathology of familial breast cancer: histological features of cancers in families not attributable to mutations in BRCA1 or BRCA2. Clin Cancer Res 2000;6:782-789.

20 Wong EM, Southey MC, Fox SB, et al. Constitutional methylation of the BRCA1 promoter is specifically associated with BRCA1 mutation-associated pathology in early-onset breast cancer. Cancer Prev Res 2011;4: 23-33.
21 The Cancer Genome Atlas Research Network. Integrated genomic analyses of ovarian carcinoma. Nature 2011;474:609-615.

22 Gilks CB, Ionescu DN, Kalloger SE, et al. Tumor cell type can be reproducibly diagnosed and is of independent prognostic significance in patients with maximally debulked ovarian carcinoma. Hum Pathol 2008;39:1239-1251.

23 Eichhorn JH, Young RH. Transitional cell carcinoma of the ovary: a morphologic study of 100 cases with emphasis on differential diagnosis. Am J Surg Pathol 2004;28:453-463.

24 Rubin SC, Benjamin I, Behbakht K, et al. Clinical and pathological features of ovarian cancer in women with germ-line mutations of BRCA1. N Engl J Med 1996;335: 1413-1416.

25 Takahashi $\mathrm{H}$, Behbakht $\mathrm{K}$, McGovern PE, et al. Mutation analysis of the BRCA1 gene in ovarian cancers. Cancer Res 1995;55:2998-3002.

26 Roh MH, Yassin Y, Miron A, et al. High-grade fimbrialovarian carcinomas are unified by altered p53, PTEN and PAX2 expression. Mod Pathol 2010;23: 1316-1324.

27 Takahashi H, Chiu HC, Bandera CA, et al. Mutations of the BRCA2 gene in ovarian carcinomas. Cancer Res 1996;56:2738-2741.

28 Werness BA, Ramus SJ, DiCioccio RA, et al. Histopathology, FIGO stage, and BRCA mutation status of ovarian cancers from the Gilda Radner Familial Ovarian Cancer Registry. Int J Gynecol Pathol 2004; 23:29-34.

29 Lakhani SR, Manek S, Frederique Penault-Llorca F, et al. Pathology of ovarian cancers in BRCA1 and BRCA2 carriers. Clin Cancer Res 2004;10:2473-2481.

30 Boyd J, Sonoda Y, Federici MG, et al. Clinicopathologic features of BRCA-linked and sporadic ovarian cancer. JAMA 2000;283:2260-2265.

31 Logani S, Oliva E, Amin MB, et al. Immunoprofile of ovarian tumors with putative transitional cell (urothelial) differentiation using novel urothelial markers: histogenetic and diagnostic implications. Am J Surg Pathol 2003;27:1434-1441.

$32 \mathrm{Wu}$ R, Hendrix-Lucas N, Kuick R, et al. Mouse model of human ovarian endometrioid adenocarcinoma based on somatic defects in the Wnt/ $\beta$-catenin and PI3K/Pten signaling pathways. Cancer Cell 2007;11:321-333.

33 Soslow RA. Histologic subtypes of ovarian carcinoma: an overview. Int J Gynecol Pathol 2008;27: 161-174.

34 Köbel M, Kalloger SE, Baker PM, et al. Diagnosis of ovarian carcinoma cell type is highly reproducible: a Trans-Canadian study. Am J Surg Pathol 2010;34: 984-993. 